\title{
TRIPLE ARTERIAL PHASE IN THE DYNAMIIC POST-CONTRASTOGRAFIC MRI STUDY OF LIVER LESIONS: COMPARATIVE STUDY
}

Curatolo Calogero ${ }^{1}$, Amato Elizabeth ${ }^{2}$, Daricello Marco ${ }^{1}$, Caruso Virginia ${ }^{1}$, Lo Re Giuseppe ${ }^{2}$, Vernuccio Federica ${ }^{1}$, Brancatelli Giuseppe ${ }^{2}$

${ }^{1}$ Dipartment of Radiology, A.O.U.P. "P. Giaccone”, Palermo

${ }^{2}$ Radiology Section, BIND, University of Palermo

KEYWORDS: Liver MRI, Triple Arterial Phase, e-TRHIVE

\section{ABSTRACT}

The objective of our work was to assess the accuracy in terms of spatial and contrast resolution, respiratory movement artifacts and synchronization of the "triple arterial phase" (TAP) sequence and compare it with the conventionally acquired arterial phase (AP). Between June 2021 and September 202116 patients underwent liver MRI for characterization and/or follow-up of focal hepatic lesions, and the TAP sequence was used. With the aim to obtain a TAP sequence we applied the KEYHOLE technique (modified to 35\%) and the CENTRA-Keyhole method (Contrast Enhanced Timing Robust Angiography) for the K-space sampling, to the basic e-THRIVE sequence. 6/16 patients had previous MRI examinations performed with the classic single AP. Five radiologists experienced in the interpretation of liver imaging independently examined MRI studies. They were asked to provide their opinion, by using a qualitative evaluation scale, on spatial resolution, contrast/noise ratio (CNR), presence of breath-hold artifacts, synchronization of the TAP and its quality and usefulness for the characterization of liver lesions. The same questions were used for the evaluation of the 6/16 previous MRI examinations acquired with the conventional AP. For all readers, with TAP, the synchronization of the arterial study, the spatial resolution, the evaluation of artifacts from incorrect breathhold were deemed good or excellent. The contrast resolution in the TAP was excellent for almost all cases. TAP was overall judged superior to AP. These preliminary results obtained are promising and require confirmation on a larger sample study to assess the diagnostic benefit of the TAP.

\section{INTRODUCTION}

Nowadays, liver magnetic resonance imaging (MRI) of the liver with intravenous (i.v.) contrast medium (CM) injection is considered the most accurate diagnostic imaging technique for the evaluation of focal liver lesions. Several studies have shown high sensitivity and specificity of liver MRI in the characterization of benign and malignant lesions and for the definitive diagnosis of hepatocellular carcinoma (HCC), allowing to reduce the need of more invasive approaches, such as liver biopsy.

The use of hepatobiliary $\mathrm{CM}$ such as gadobenic acid (Gd-BOPTA) and gadoxetate disodium (GdEOB-DTPA), exploiting the specific absorption by hepatocytes during the hepatobiliary phase, provides a high signal contrast between liver and hepatocytefree lesions, helping in the differential diagnosis between HCC and other liver lesions as well as in the detection of small HCCs, as outlined by numerous scientific studies.

However, proper synchronization of the hepatic arterial phase remains a fundamental and often essential factor for the detection and characterization of HCC.

Indeed, the detection of lesion enhancement in the arterial phase is mandatory to reach the radiological diagnosis of HCC.

The growing technological advances in the field of MRI, including the development of increasingly performing MRI scanners in terms of amplitude and slew-rate, the introduction of ever more fast sequences, the development of more and more efficient and robust parallel omaging techniques that enable ever higher $\mathrm{R}$ acceleration factors without incurring in typical artifacts from subsampling K-dataspace and, finally, the innovative corrections of motion artifacts from an incorrect breath-hold until the introduction of AI software, have led to exponential improvements and optimizations of imaging in MRI.

However, in liver MRI imaging capturing an "adequate" arterial phase has always been a challenge due to the narrow temporal window, which does not allow margin for error.

\section{OBJECTIVES}

The purpose of our study was to describe a new sequence, recently introduced in our Radiology Unit, the Rapid-Acquisition GRE T1 3D Spoiled FidImaging, to obtain a "triple arterial phase" (TAP), which allows to obtain three phases, in a single overall acquisition of 30 seconds, thus allowing to cover the temporal window of the hepatic arterial phase.

The main objective of our work was to assess the accuracy in terms of spatial resolution and contrast, respiratory movement artifacts and synchronization of the TAP and compare it with the conventionally acquired arterial phase, in those patients who had previous MRI examinations performed without TAP.

\section{MATERIALS AND METHODS}

\section{Study population}

Between June 2021 and September 202116 patients 
(12 males and 4 females aged 29-82 years, average age 60 years) underwent liver MRI with the sequence Rapid-Acquisition GRE T1 3D Spoiled Fid-Imaging for characterization and/or follow-up of one or more focal hepatic lesions.

Of these patients, 6 out of 16 had previous MRI examinations performed with the classic single arterial phase at our Department or in other radiological structures.

MRI examinations were performed with a high-field MRI scanner, 1.5T Philips Achieva equipped with gradients combined with amplitude of $30 \mathrm{mt} / \mathrm{m}$ and Slew-Rate of $80 \mathrm{mt} / \mathrm{m} / \mathrm{ms}$. The TORSO XL surface coil 16 channels (Phased-Array) was used for the liver MRI studies. The surface coil is positioned directly on the area of interest, allowing to take full advantage of the signal to noise ratio/coil distance. It is a receiving coil only while the RF pulse is transmitted by the Q-Body coil.

\section{MIRI EXAMINATION TECHINIQUE}

The study protocol and the correct choice of pulse sequences play an important role in the characterization of focal liver lesions. The protocol allows the evaluation of liver parenchyma in the unenhanded phase (without CM administration) and in the postcontrast phases, i.e. arterial, portal venous and delayed phases, as well as in the hepatobiliary phase in case of adoption of Gd-BOPTA or Gd-EOBDTPA as CM.

The examination requires a close collaboration of the patient to allow the acquisition of images with good resolution, high diagnostic content and, above all, lack of artifacts due to inadequate breath-hold. For this reason, patients preparing to perform a liver MRI must be well-educated on respiratory apnea techniques, during which the scanner will collect complex data related to magnetizations $\mathrm{Mx}$ and $\mathrm{My}$ (conventionally).

Other sequences will also be performed through the patient's respiratory synchronization (respiratory triggered sequences). The latter technique is very robust but requires more time than the breath-hold technique. All the patients have been studied in supine decubitus with the arms placed (preferably) over the head, making sure that the hands do not touch, so as to avoid closed circuits.
After having acquired the "Survey" or "Localizer" sequence (usually a Balanced-TFE sequence), that allows to establish the study range for the subsequent sequences, the evaluation of the hepatic parenchyma involves the acquisition of the following sequences:

- SE Single-Shot High-Speed highly T2 weighted, known as SSFSE (Philips and GE), HASTE (Siemens) or FASE (Toshiba/Canon) without and with fat suppression, using SPAIR method, performed on the axial and coronal plane (optional). A further similar sequence but with higher TE (TE 300) is acquired to facilitate discrimination between e.g. cysts and angiomas.

- GRE Multi-echo Multi Contrast Fid-Imaging T1weighted, known as DUAL FFE In/Out (Philips), FLASH In-Out Phase (Siemens), GRE In-Out Phase (GE), Dual Echo GRE (Toshiba/ Canon). The main application of this sequence is to identify the intracellular fat content by showing signal drop on "Out of Phase" images, which can be intralesional in some HCCs.

- SE-EPI DWI (Diffusion Weighted Imaging) in order to evaluate the restriction of the diffusivity of water molecules in lesions.

The sequence GRE Rapid Acquisition 3D T1-spoiled Fid-Imaging with fat suppression using SPAIR method (evolution involves the DIXON method), known as e-THRIVE (Philips), VIBE (Siemens), LAVA (GE), Quick-3D (Toshiba/Canon) represents the sequence used for the dynamic post-contrast study of the liver. In particular, in our study, the classic e-THRIVE sequence underwent several applications in order to greatly accelerate the single apnea, thus reducing the timing for sequence acquisition from 17-20 seconds of the classic e-THRIVE to 6-7 seconds per single phase of triple arterial phase (TAP).

In detail, to the basic e-THRIVE sequence, already equipped with acceleration techniques such as Parallel Imaging (SENSE), Zero Filling Interpolation, and Half Fourier Phase Encoding, we applied the KEYHOLE technique (modified to 35\%) and the CENTRAKeyhole method (Contrast Enhanced Timing Robust Angiography) for the K-space sampling.

In this way, the $\mathrm{K}$-space is divided into several elliptical or annular regions that are sampled according to the key-hole method and the association of the VIEW-

\begin{tabular}{|c|c|c|c|c|c|}
\hline Question & $\begin{array}{c}\text { Pt. 1 } \\
\text { Insufficient }\end{array}$ & $\begin{array}{c}\text { Pt 2 } \\
\text { Mediocre }\end{array}$ & $\begin{array}{c}\text { Pt 3 } \\
\text { Sufficient }\end{array}$ & $\begin{array}{c}\text { Pt 4 } \\
\text { Good }\end{array}$ & $\begin{array}{c}\text { Pt 5 } \\
\text { Excellent }\end{array}$ \\
\hline Q1 & 0 & 0 & 0 & 7 & 9 \\
\hline Q2 & 0 & 0 & 0 & 3 & 13 \\
\hline Q3 & 0 & 0 & 0 & 6 & 10 \\
\hline Q4 & 0 & 0 & 0 & 2 & 14 \\
\hline Q5 & 0 & 0 & 0 & 2 & 14 \\
\hline
\end{tabular}

Tab.1- Qualitative evaluation of the 16 sequences acquired in "Triple arterial phase" in 16 patients.

\begin{tabular}{|c|c|c|c|c|c|c|}
\hline Question & $\begin{array}{c}1 . \\
\text { TAP/AP }\end{array}$ & $\begin{array}{c}2 . \\
\text { TAP/AP }\end{array}$ & $\begin{array}{c}3 . \\
\text { TAP/AP }\end{array}$ & $\begin{array}{c}4 . \\
\text { TAP/AP }\end{array}$ & $\begin{array}{c}5 . \\
\text { TAP/AP }\end{array}$ & $\begin{array}{c}6 . \\
\text { TAP/AP }\end{array}$ \\
\hline Q1 & $5 / 4$ & $4 / 4$ & $4 / 3$ & $5 / 4$ & $4 / 5$ & $4 / 5$ \\
\hline Q2 & $5 / 5$ & $5 / 4$ & $4 / 4$ & $5 / 4$ & $4 / 4$ & $5 / 5$ \\
\hline Q3 & $4 / 3$ & $4 / 1$ & $5 / 3$ & $4 / 3$ & $5 / 4$ & $5 / 5$ \\
\hline Q4 & $5 / 3$ & $5 / 1$ & $5 / 4$ & $5 / 3$ & $5 / 4$ & $5 / 5$ \\
\hline Q5 & $5 / 4$ & $5 / 4$ & $5 / 4$ & $5 / 4$ & $5 / 3$ & $5 / 5$ \\
\hline
\end{tabular}

Tab. 2 - Comparison between the triple arterial phase (TAP) and the conventional single arterial phase (AP) per 


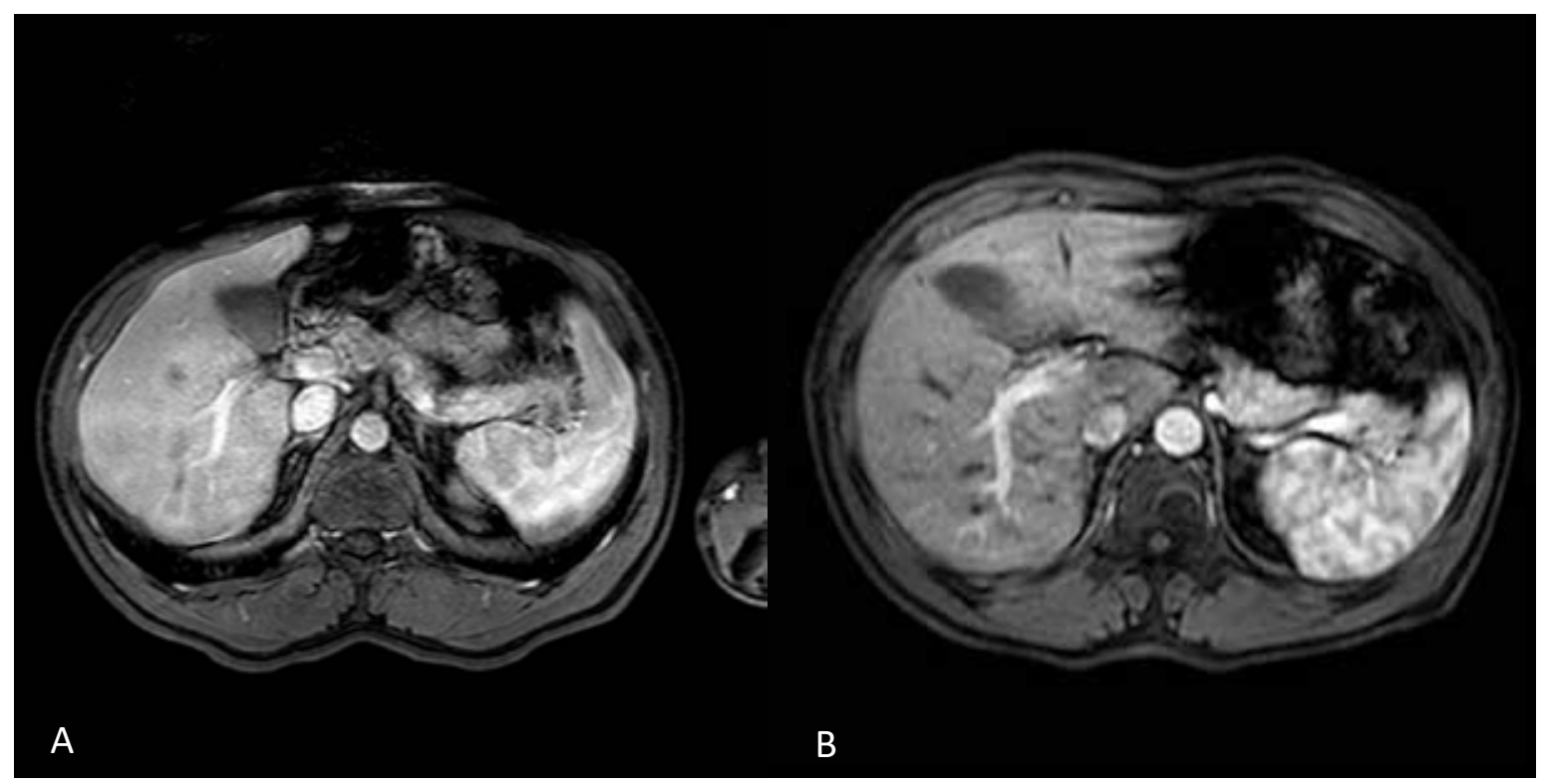

Fig. 1 - 48-year-old male with genetic predisposition to hereditary tumors..Comparison in terms of contrast resolution, spatial resolution and arterial phase synchronization between the classic AP (A) and the Triple arterial phase (B).

SHARING technique allows to obtain the overall and over time data sharing of the individual three phases. Moreover, since the duration of respiratory apnea to include all the three phases of the TAP would have been inadequate, we inserted between each phase of the TAP the "Manual Start" necessary to prepare the patient $(1-2 \mathrm{sec})$ to the next breath-hold of the subsequent phase thus avoiding the artifacts due to breath-hold.

In all the examinations, the beginning of the acquisition of the TAP was based on fluoroscopy MRI (Bolus-Trak) with monitoring of the aorta: the acquisition of the TAP begins when the CM begins to arrive in the descending aorta.

\section{ANALYSIS OF IMAGES}

Five radiologists experienced in the interpretation of liver imaging independently examined MRI studies. They were asked to provide their opinion on spatial resolution, contrast/noise ratio (CNR), presence of breath-hold artifacts, synchronization of the TAP and its quality and usefulness for the characterization of liver lesions.

For this purpose a qualitative evaluation scale was used with scores from 1 to 5: 1 (insufficient), 2 (mediocre), 3 (sufficient), 4 (good) and 5 (excellent). The same questions and scale were used for the evaluation of the six out of 16 previous MRI examinations acquired with the conventional single arterial phase (AP), for comparative purposes.

\section{RESULTS}

Table 1 shows the evaluations of the five independent radiologists on the TAP sequence obtained in 16 liver MRI studies, according to a 5-point qualitative scale, on the following questions: spatial resolution (Q1), contrast resolution (Q2), incorrect breath-hold artifact (Q3), arterial phase synchronization (Q4), overall quality for characterization of liver lesions (Q5).

Table 2 summarizes the evaluation of the TAP and conventional AP in the 6 patients who had at least a previous MRI examinations acquired with the conventional AP.

\section{formed in previous MRI studies in 6/16 patients.}

Fig. 1 and 2 show two comparative MRI examinations, one with the acquisition of the classical AP and one with acquisition of TAP.

\section{DISCUSSION}

In this study, all readers confirmed the reliability and efficiency of the triple arterial phase in the synchronization of the arterial study with a good or excellent evaluation in $13 \%$ and $87 \%$ of the exams, respectively. We obtained similar results in regard to the evaluation of the overall quality for the characterization of hepatic lesions, thus proving the importance of TAP for diagnostic purposes.

Although limited by the low number of patients included, our results confirm the likely superiority of the triple arterial phase over the single arterial phase, especially in terms of synchronization, that was superior in $5 / 6$ with TAP compared to conventional AP.

In particular, the synchronization of the single AP was judged insufficient in $1 / 6$ and sufficient in $2 / 6$.

Although the contrast resolution in the Triple Arterial phase was excellent for almost all cases, with a good rating in only $3 / 16$ cases, we found no differences compared to the classic e-THRIVE.

This technique does not limit the sampling of the central lines ("useful" data responsible for contrast resolution) of k-space, but it samples them more frequently, ensuring equal performance in contrast resolution despite shorter acquisition times.

Spatial resolution was deemed excellent in about $56 \%$ of cases and good in 44\%, with a lower qualitative assessment compared to the classic e-THRIVE in $2 / 6$ cases. The explanation for this result lies in the reduced time available to acquire k-space data and in the k-space sampling mode of the Key-hole type which inevitably results in a reduction of peripheral data sampling (spatial resolution data).

In the evaluation of artifacts from incorrect breathhold, the TAP was excellent in 10/16 cases and good in 6/16. In the comparative analysis, TAP was always higher than the AP. The explanation is clearly 


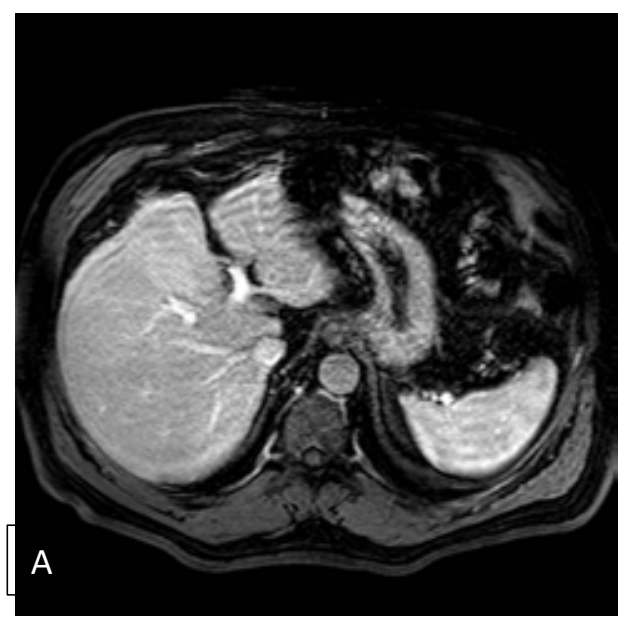

Fig. 2 - Follow-up for HCC in a 63 year old male patient. In the previous RM examination performed with classic arterial phase obtained with the conventional AP (A), there are artifacts from incorrect Breath-Hold and synchronization with the hepatic arterial phase was not optimal; indeed, $\mathrm{CM}$ is in the hepatic veins, thus indicating that the AP is inadequate (delayed AP). In the MRI examination performed with the Triple Arterial Phase (B1,B2,B3) the second phase (B2) appears to meet the requirements of an adequate hepatic arterial phase, with lack of artifacts and optimal spatial and contrast resolution.

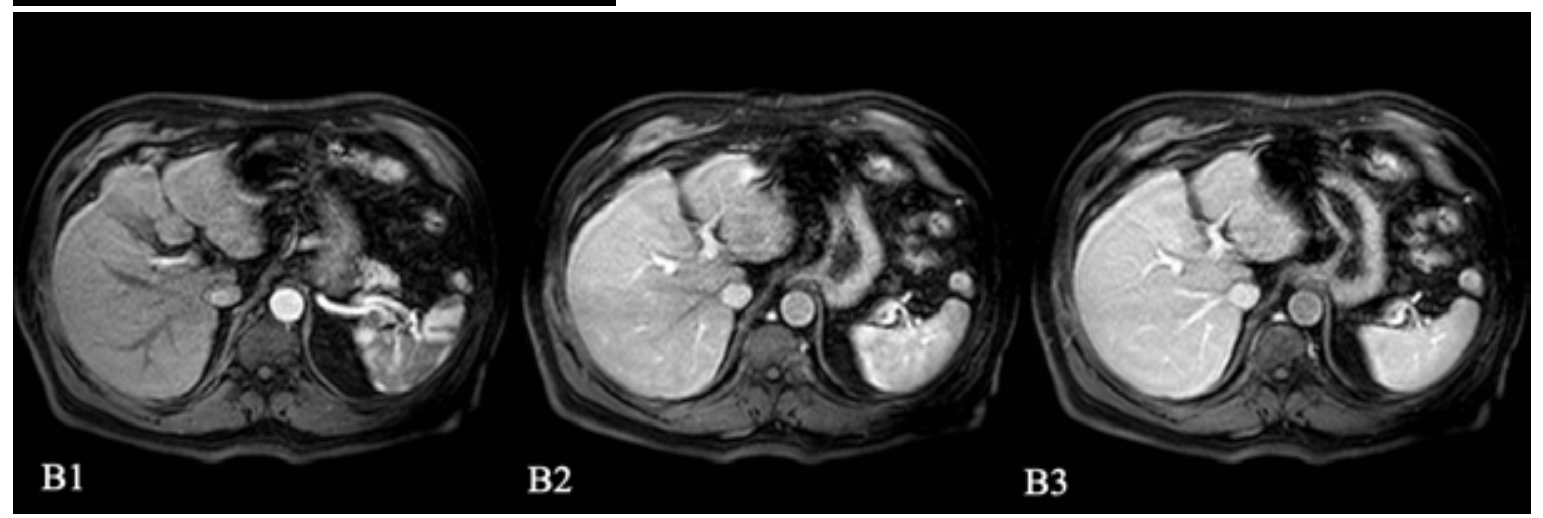

attributed to the different duration of the single respiratory apnea, that is about $17-20$ seconds for the classic e-THRIVE compared to 6-7 seconds of each phase in the TAP.

The preliminary results obtained are promising and require confirmation on a larger sample, assessment of the learning curve for MRI radiographers, assessment of inter and intra-reader evaluation of TAP as well as a clinical study on focal liver lesion to assess the diagnostic benefit of the TAP overall and with gadoxetate disodium specifically, as conventional
AP with gadoxetate disodium is often hampered by motion artifacts.

\section{CONCLUSIONS}

In our pilot study the triple arterial phase obtained by modifying the classic E-THRIVE allowed to obtain a high temporal resolution for acquisition of three arterial phases able to cover the entire temporal window of a single hepatic arterial phase without significant breath artifacts.

\section{REFERENCES}

1. Bhandari R, Kirilina E, Caan M, Suttrup J, De Sanctis T, De Angelis L, Keysers C, Gazzola V. Does higher sampling rate (multiband + SENSE) improve group statistics - An example from social neuroscience block design at 3T. Neuroimage. 2020;213:116731. doi: 10.1016/j.neuroimage.2020.116731.

2. David J Larkman, Joseph V Hajnal, Amy H Herlihy, Glyn A Coutts, Ian R Young, and Go sta Ehnholm, "Use of multicoil arrays for separation of signal from multiple slices simultaneously excited," Journal of Magnetic Resonance Imaging, vol. 13, no. 2, pp. 313-317, 2001.

3. McElroy S, Ferrazzi G, Sohaib Nazir M, Kunze K.PK, Neji R, Speier P,Stäb D, Forman C, Reza Razavi R Chiribiri A , Roujol S. Combined simultaneous multislice bSSFP and compressed sensing for first-pass myocardial perfusion at 1.5 T with high spatial resolution and coverage Magnetic Resonance in Medicine Received: 28 January 2020 Revised: 14 May 2020, Accepted: 14 May 2020 DOI: $10.1002 / \mathrm{mrm} .28345$

4. Yoon H, Dong-wook L, Lee L, Choi SH, Sung-Hong Park and Jong Chul Ye Multiband Dynamic Compressed Sensing 2015 IEEE 12th International Symposium on Biomedical Imaging (ISBI) Electronic ISBN: 978-1-4799-2374-8 INSPEC Accession Number: 15309786 DOI:10.119/ISBI.2015.7164021 